\title{
Image-based reconstruction of informal settlements
}

\section{Conference Paper}

Author(s):

Mason, Scott; Baltsavias, Emmanuel P.

Publication date:

1997

Permanent link:

https://doi.org/10.3929/ethz-a-004333536

Rights / license:

In Copyright - Non-Commercial Use Permitted

Originally published in:

https://doi.org/10.1007/978-3-0348-8906-3_10 


\title{
Image-Based Reconstruction of Informal Settlements
}

\author{
Scott Mason* and Emmanuel Baltsavias** \\ *Department of Surveying \\ $\&$ Geodetic Engineering \\ University of Cape Town \\ Rondebosch 7700, South Africa \\ Ph.: +27-21-650 3574 \\ Fax: +27-21-650 3572 \\ mason@engfac.uct.ac.za \\ **Institute of Geodesy \&Photogrammetry \\ ETH-Hoenggerberg \\ CH-8093 Zurich, Switzerland \\ Ph.:+41-1-633 3042 \\ Fax: +41-1-633 1101 \\ manos@geod.ethz.ch
}

\begin{abstract}
The improvement of living conditions in informal settlements (IS) is one of the most complex and pressing challenges facing developing countries. In this paper we report on progress towards the automated generation of geospatial databases of informal settlements from large scale, low cost, digital still-video imagery. We focus on shack extraction as the predominant data requirement. In contrast to western residential environments where many structures are geometrically complex yet texturally regular, sample studies in informal settlements suggest that up to $90 \%$ of shacks are approximately rectangular in shape with a flat roof, but texturally complex due to the diverse construction materials. We present a strategy for automating the detection and extraction of these structures. Preliminary results for different cues are discussed. Central to this strategy is the integration of cues in map (object) space. First experiences suggest a framework based on attributed contours, 2.5D blobs derived from digital surface models and extracted shadows will suffice for modeling shack outlines plus a representative height.
\end{abstract}

\section{Introduction}

Informal settlements (also known as "squatter settlements" or "shanty towns") may be defined as dense settlements comprising communities housed in self-constructed shelters under conditions of informal or traditional land tenure (Hindson and McCarthy, 1994). They are a common feature of developing countries (DC) and are typically the product of an urgent need for shelter by the urban poor. As such, they are characterized by a dense proliferation of small, make-shift shelters built from diverse materials (e.g. plastic, tin and asbestos sheeting, wooden planks), degradation of the local ecosystem, e.g. erosion, poor water quality and sanitation, and severe social problems. A UNCHS global report on human settlements in 1986 pointed out that between 30 and 60 percent of residents of most large cities in DC live in informal settlements. South Africa is not spared this situation. In the Cape Town area alone there are estimated to be some 120 IS.

In the developed world developments towards technologies for reconstructing 3D models of urban environments are driven by applications such as architectural planning, illumi- 
nation design and even virtual tourism (Mason and Streilein, 1996). Spatial models of informal settlements, in contrast, are required to support efforts to improve living conditions. Applications in which spatial data play an important role range from monitoring IS growth at the regional level to the management of individual settlements, to shack counting for electoral boundary determination, the generation of GIS/CAD databases of IS infrastructure (huts, tracks, water outlets, etc.) for service upgrading, soil and ground water quality evaluation for environmental quality assessment, and settlement upgrade scenario modeling. With the exception of settlement upgrading, requirements for spatial models of IS emphasize less the need for positional and object modeling accuracy and more completeness of records in the face of highly dynamic environments. In all cases, integration of a complete settlement spatial model with socio-economic data in a GIS environment is considered a major facilitating factor towards improved IS management (Mason and Rüther, 1997).

To date, the spatial modeling of IS has been carried out using conventional, mostly photogrammetric, mapping techniques. Numerous authors and agencies have promoted mapping methods better suited to the low-cost/low-tech DC situation, e.g. small-format aerial imagery. The UNCHS (Habitat), in particular, has developed the Visual Settlement Planning (ViSP) IS mapping and planning methodology based on PC-based GIS tools using data manually digitized from scanned medium-format aerial imagery. The objectives of the UrbanModeler project in the Department of Surveying and Geodetic Engineering, University of Cape Town, are to improve upon manual methods with the development of reliable methods and computer-based tools for automated settlement mapping. The research reported here stems from collaborative research between UCT and ETH Zurich.

This article outlines the needs and complexities of generating spatial models of IS. It reports on first experiences in developing a strategy for automated shack extraction. Key features of this strategy are its operation in object space enabling integration in a desktop GIS environment.

\section{Informal Settlement Spatial Modeling Requirements}

\subsection{Image Analysis Tasks}

Given the dynamics of IS, their density and the type and quantity of spatial data required for management, imagery is clearly a major source of data. The roles of imagery and image analysis in supporting these applications include:

- Detection of settlements e.g. regular satellite-image based control of urban areas.

- Detection of infill and monitoring of change in settlement boundaries, density etc.

- DTM (Digital Terrain Model) and DSM (Digital Surface Model) generation.

- Ground cover classification for environmental quality assessment.

- Detection of shacks, e.g. shack counting in support of social surveys.

- Detail settlement mapping including the extraction of shacks, other buildings, 
tracks, services in support of geospatial model generation.

- Reconnaissance and response to emergencies.

In addition to these modeling roles image media can play a key role in communicating spatial information to IS residents. In South Africa at least, perceptions of space and spatial relations differ markedly between African and European cultures. Line maps are often not well understood.

In this work we focus firstly on the task of rapid "first time" settlement mapping. A second, and in the long term more important thrust, is the detection and updating of change in IS geospatial models. The ability to accurately monitor the dynamics of an IS is critical in ensuring informed decision-making. We focus initially on the detection and extraction of shacks from digital aerial imagery given that shacks are the primary spatial unit in IS and constitute the link between the spatial and the non-spatial (socio-economic data are usually collected at shack level).

\subsection{Image Sources}

Detailed mapping of informal settlements has traditionally been carried out photogrammetrically using large-format aerial photography. Data is compiled using analogue or analytical (depending on local technology resources) methods, i.e. manually, labourintensive and hence slow, requiring considerable expertise and expensive equipment. These methods are uneconomical over the relatively small, if dense, areas covered by informal settlements, particularly for frequent updating. Alternative imaging sources and rapid mapping techniques are therefore needed.

Of low-cost imaging devices, digital cameras are preferred due to the direct capture of digital data. Currently, however, these cameras do not match their analogue counterparts in resolution. Moreover, slow image download rates can lead to more complex flight planning. Multiple runs of individual strips are needed to provide for overlapping imagery at large image scales. Nevertheless, because the areal extent of many informal settlements is moderate, these limitations can be practically overcome in a large number of situations, e.g. as was demonstrated in a mapping campaign of the Marconi Beam settlement using a Kodak DCS460c digital camera (Mason et al., 1997). Preliminary triangulation results indicate planimetric and elevation accuracies in the order of $20 \mathrm{~cm}$ and $40 \mathrm{~cm}$ respectively, from the 1: 18500 scale imagery. This is sufficient for most IS applications including 1: 2000 mapping with $0.5 \mathrm{~m}$ contours. Further testing is needed to verify these results under a range of conditions. The test data used in this work is drawn from this work.

Based on practical experiences desirable characteristics of imagery for IS detail mapping which influence the interpretability of IS scenes and provide useful cues for automated feature extraction include:

- Large image scale and high resolution. The minimum ground pixel size in digital imagery should be in the order of $0.5 \mathrm{~m}$. Finer resolution is needed for reliable mapping of communal toilet facilities, services such as water outlets, etc. 
- Colour imagery is preferred to improve interpretability.

- Flying times should be chosen to ensure strong shadows. Shadows are useful in manual scene interpretation as well as in automated shack extraction (see Sec. 4.6).

- Nadir stereo imagery with high overlap is required for DSM generation.

Ground control is somewhat problematic given that signalization materials are likely to be rapidly converted into roofing. Roof corners of shacks are often suitable and can be rapidly coordinated using kinematic GPS fixing. This must be performed immediately after flight to avoid gross errors introduced by shacks "renovations". More substantial community buildings are therefore preferred.

\section{Automated Shack Extraction Strategy}

Unlike residential environments in developed countries development patterns in IS do not follow regular design codes but are subject to a complex interplay of natural (terrain, environmental), social (population pressures), historical and political forces. It is therefore impossible to define a narrow contextual description. In this Section we first investigate shack characteristics with view to the identification of reliable extraction cues and use these to formulate a strategy for automated shack extraction.

\subsection{Shack Characteristics}

IS are typically characterized by a mix of primarily informal and a few formal buildings (play schools, community centres). The majority of buildings are shacks with the following properties:

- Single storied structures with flat and near-horizontal roofs. A small percentage of shacks are multi-storied and/or hip-roofed.

- Simple geometry. A study of shack shape typology in the Marconi Beam settlement in Cape Town showed that $85 \%$ of shacks are 4-sided and $11 \%$ 6-sided. Deviations from rectangularity up to 30 degrees are, however, not uncommon.

- Shack dimensions range from $4 \mathrm{~m} \times 4 \mathrm{~m}$ with roof heights between 2 - 2.5m.

- Constructed from diverse materials (e.g. plastic, iron sheeting, timber) with variable textures and colours even for a individual shack.

- Often very densely built, e.g. 2 - 3m separation.

- Situated in variable contexts although commonly characterized by a general lack of vegetation.

A lack of regularity in most shack properties implies that many of the vision cues commonly used in building extraction in other domains are less reliable for use in IS reconstruction. For example, geometrical constraints such as parallelity and orthogonality cannot be strongly imposed. Similarity measures such as those based on photometric and chromatic attributes, as used in Henricsson et al. (1996) will also be less reliable due to mixed building materials. 


\subsection{Considerations}

In this section we outline a strategy for shack extraction from large-scale aerial imagery. The following considerations apply:

- Automation. Recognizing that full automation is not attainable we aim to reduce the complexity of the shack reconstruction task by concentrating on classes of shacks most likely to be extractable and embedding the strategy in an interactive environment.

- Initial focus on "first time" mapping.

- Shack modeling: 2D roof outline and a sample height.

- Low-cost image acquisition: small format film, still video CCD, and standard \& digital video are preferred for practical reasons. The desirable image characteristics listed in Sec. 2.2 are assumed.

- Integration in a desktop GIS environment. This should enable exploitation of standard functions for the I/O, management, spatial analysis, editing, visualization and fusion of hybrid data. It also aims at enhancing the accessibility of tools developed to end users. To this end, the GIS software should be low-cost, a market standard and expandable. Stereo display should not be a requirement for data extraction although it may be desirable for supporting interpretation.

- Simplicity. Accessibility to low skilled operators is emphasized. Ideally, local knowledge integrated can be exploited by harnessing IS residents as part of selfhelp community capacity development projects (Mason and Rüther, 1997).

Integration in a desktop GIS environment implies performing shack extraction in object space. Orthoimages therefore form the image source employed. The advantage of object space reconstruction is that all geocoded sources of information, e.g. from earlier mapping epochs, can be directly exploited. Moreover, as desktop GIS nowadays support orthoimages and polygon data capture tools, interactive data capture functions already exist and can be built upon. For historical reasons we base our work on the PC ARC/ INFO and ArcView desktop GIS products. We are integrating our shack extraction functionality into these tools using AMLs and Avenue scripts. PC-based Erdas Imagine is used for image processing tasks such as multispectral classification.

\subsection{Shack Extraction Strategy}

Based on the above-mentioned considerations the strategy for shack extraction shown in Figure 1 is proposed. This strategy is based on the following principles:

- Automate extraction of the simplest shacks, e.g. 4-sided shacks. As this is the most common shack form reliable automated extraction of a high percentage of this class of shacks should deliver improvement in the efficiency of geospatial database generation.

- Multiple cues (e.g. edge contours, shadows, DSM etc.) are fused in a two-step procedure of shack detection followed by shack extraction. 
- Manual support for the automated procedure, e.g. for complicated roofs and/or where cues are inadequate, is accommodated in the shack extraction step.

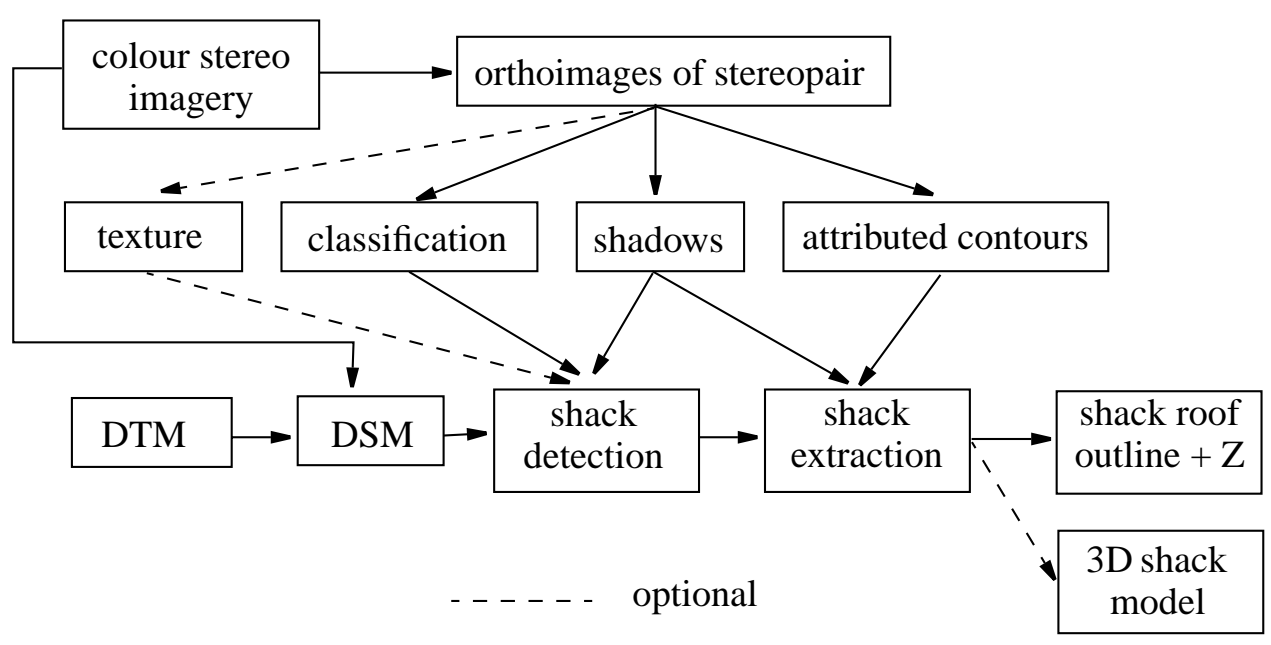

Figure 1: Shack reconstruction strategy.

The shack reconstruction strategy assumes the existence of a detailed DTM of the area being modeled, e.g. derived from an earlier conventional photogrammetric survey. This assumption, which has wide validity given that the terrain is stable over time and IS development will have limited impact on it, enables image analysis to focus on orthoimages derived from a DTM. This has the advantages of simplifying the integration of cues from multiple sources and the output is in a form suitable for direct dissemination to planners, etc. Results from automated shack extraction procedures may be overlaid on the orthoimages and shacks corrected in manual fashion. The overlay capacity is of particular advantage in IS update mapping; only changes to previous epoch shack outlines need to be mapped.

\section{Evaluation of Information Sources}

\subsection{Test Imagery}

One of the goals of this work is to evaluate low-cost imaging sources for IS mapping. A test data set was derived from a stereopair of DCS460 imagery from the pilot study referred to in Sec. 2.2. Ground truth for this set was produced by manual shack measurement in the orthoimages in ArcView 3 (see Figure 2). It was measured monoscopically in one of the orthoimages although in some difficult cases viewing with a Leica DVP stereoviewing system was used. Note that inaccuracies in the planimetric positions of shacks extracted from single orthoimages due to their non-inclusion in the DTM are insignificant for most IS applications. 


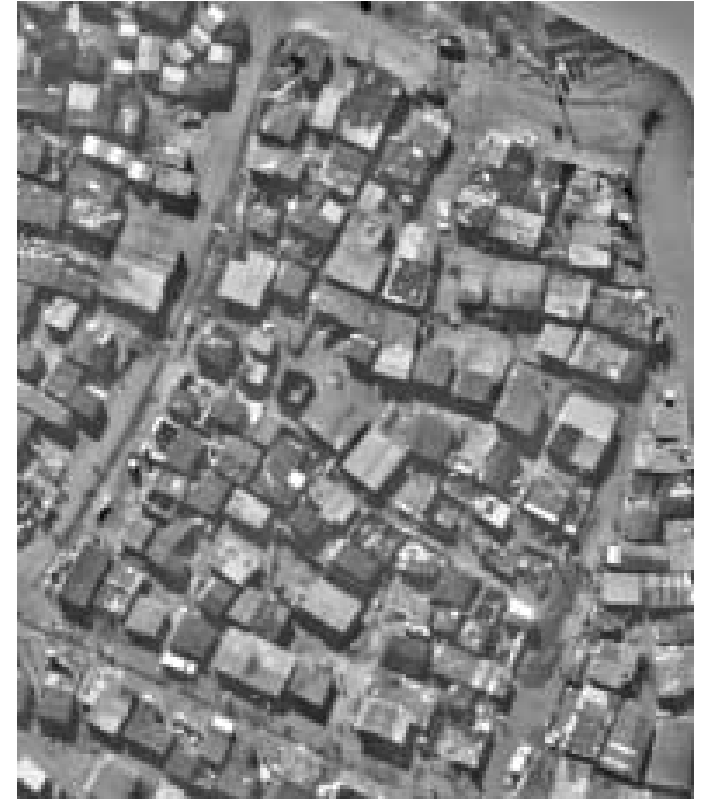

(a)

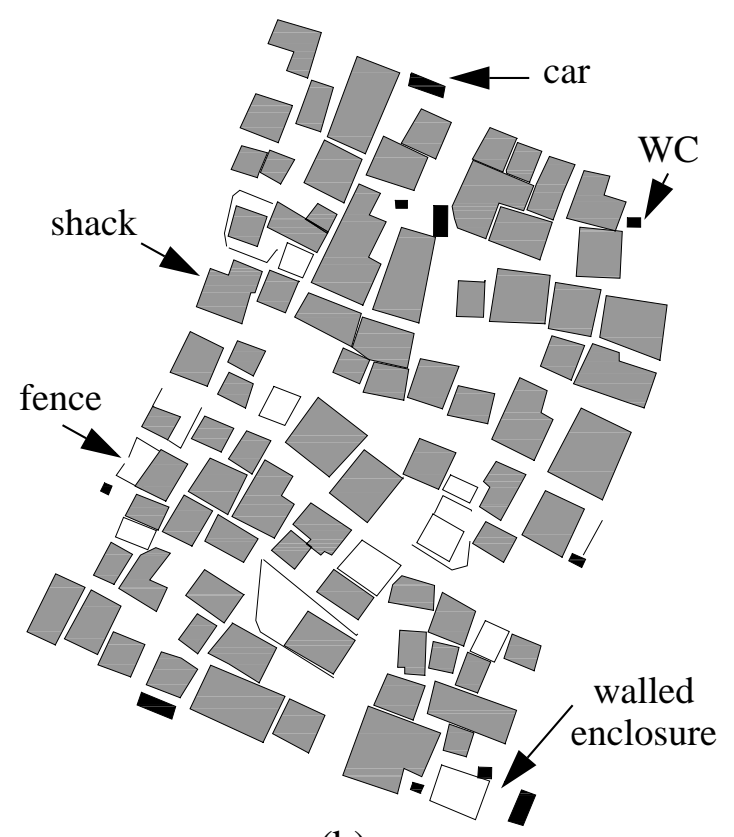

(b)

Figure 2: Test data and ground truth: (a) Wallis filter enhanced section of orthoimage; (b) corresponding ground truth data showing above-surface man-made structures.

\subsection{Multispectral Classification and Shadows}

The potential of multispectral classification to support man-made object (MMO) extraction in digital imagery is well-recognized, however to date it has found only limited application. For example, Henricsson et al. (1996) show that for residential scenes the spectral signatures of vegetation and MMO features in scanned false-colour IR imagery are distinct enabling reliable classification into fore- and background. In IS, however, the "background" is largely bare ground which is spectrally similar to many shack materials in RGB as well as in false-colour IR imagery.

Figure 3 illustrates the results of multispectral classification on the test imagery (see Li and Mason (1997) for a more detailed analysis). Figure 3a shows a scattergram of the two bands after principal component analysis (PCA) on the original enhanced RGB imagery. The ellipsoids represented the $2 \sigma$ variance of 5 object classes about their mean values. These classes were derived from a process of unsupervised classification to determine 10 statistically separable classes followed by merger of these classes into 5 identifiable object classes shadows, ground and three types of shack roofs dark, medium and bright in a supervised classification step. The denotation of the roof classes reflects the fact that their spectral distinction lies more in differing luminance than in chromatic variation. Figure $3 \mathrm{~b}$ illustrates the result of merging the 3 shack (light) and 2 non-shack (dark) classes. Examination of this result reveals the difficulties of shack-ground separation. Shack hypothesis generation based on multispectral classification alone leads to an unacceptable number of false alarms. Similarly, many shacks are partially classified as ground. 


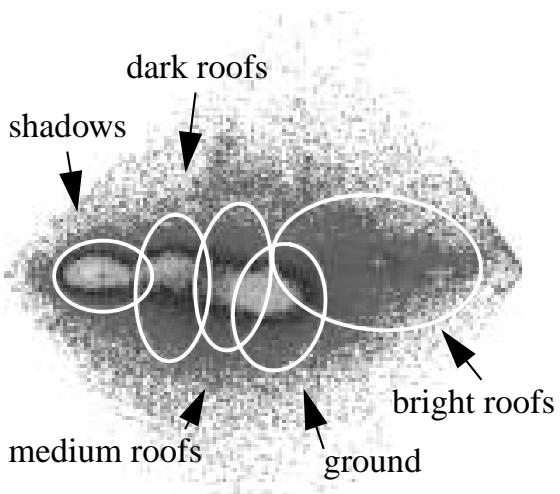

(a)

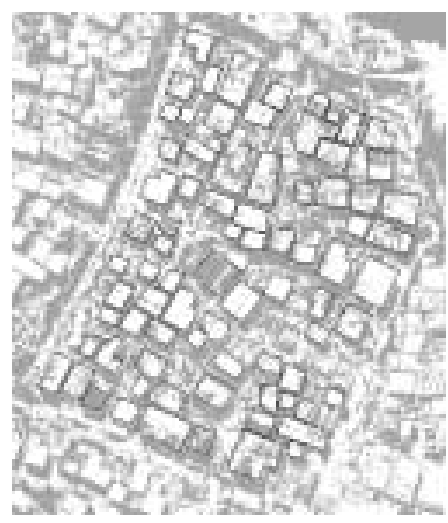

(b)

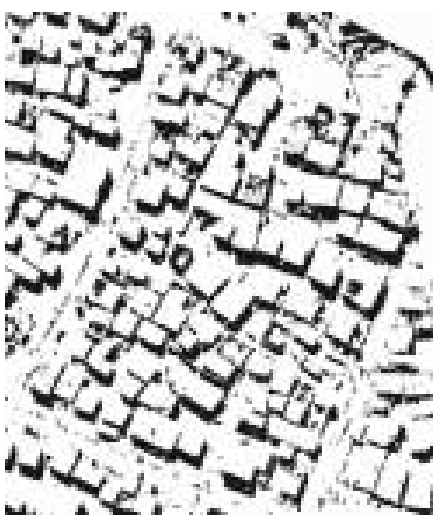

(c)

Figure 3: Results of multispectral classification: (a) scattergram of PCA bands 1 and 2 overlaid with IS object class ellipsoids ( $2 \sigma$ level); (b) combined shack classes (white) overlaid with ground truth data; (c) shadow class (black).

\subsection{Shadows}

The scattergram in Figure 3a suggests that the class shadows is largely distinct of other object classes. This is confirmed by Figure $3 \mathrm{c}$ showing the result of multispectral classification of the image in Figure 2a into shadows and non-shadows. An equivalent result can be achieved from segmentation of gray-level images. Importantly, with few exceptions the shadows have been reliably classified. This result presents a number of shack detection and extraction possibilities, as detailed below.

\subsection{Digital Surface Model}

Dense DSMs have been shown to be useful in both the detection of buildings, i.e. as blobs on the terrain, and in building reconstruction (Baltsavias et al., 1995; Henricsson et al., 1996). Note that DSMs cannot be used for orthoimage generation due to smearing effects resulting from the imprecise modeling of MMOs. For these investigations DSMs were generated using an implementation of Geometrically Constrained Least Squares Image Matching. Exterior and interior orientation values were adopted from the triangulation study reported in Mason et al. (1997). A number of different tests were conducted using combinations of preprocessing (none, Wallis filtering and median filtering) and match point selection (image space grid and interest points selected using an interest point operator). Best results were obtained using image space grid point generation on the Wallis filtered images (see Figure 4a).

Blob detection using the DSM was performed in two steps: (1) by producing the normalized DSM, i.e. DSM minus DTM; (2) thresholding of the normalized DSM at $1.5 \mathrm{~m}$. This threshold was chosen so as not to exclude the lowest shacks accounting for some smoothing in the DSM peaks, but to exclude possible surface features, such as cars. Figure $4 \mathrm{~b}$ shows that all shacks have been at least partially included in the extracted blobs. It 
is evident, however, that many shack blobs are connected, thus leading to difficulties in individual shack detection. This problem can be largely overcome by elimination of blob regions that overlay shadow regions. Figure $4 c$ shows the result of masking the DSM blobs in Figure $4 \mathrm{~b}$ with the shadows in Figure $3 \mathrm{c}$. The shadow-refined shack blobs can be used to hypothesize shacks. Cleaning of this result is required to remove small, nonshack objects. Verification of blobs as shacks, e.g. using image texture measures (see Baltsavias et al., 1995), would need to be employed in areas where vegetation exists. It is important to note that the (coarse) delineation of shacks by the resultant 2.5D blobs suffices as an end result for many IS applications, including shack counting.

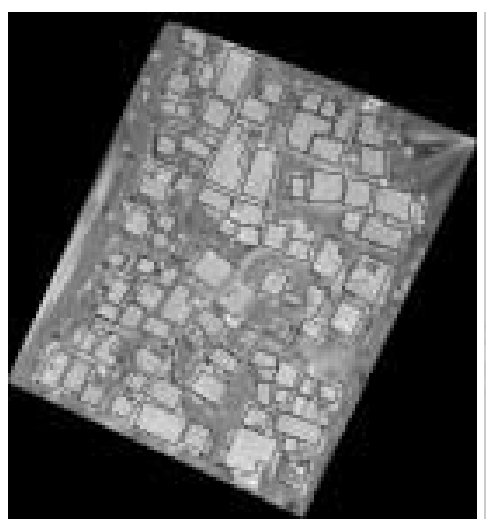

(a)

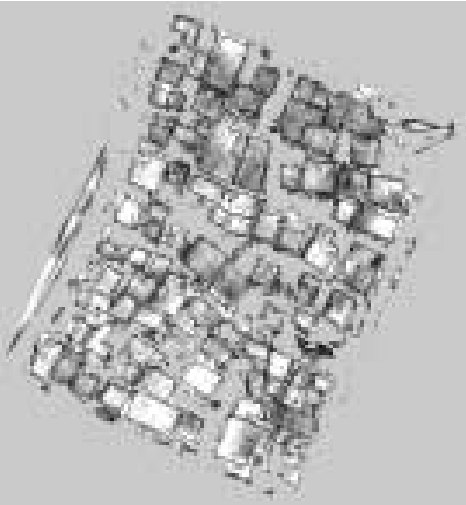

(b)

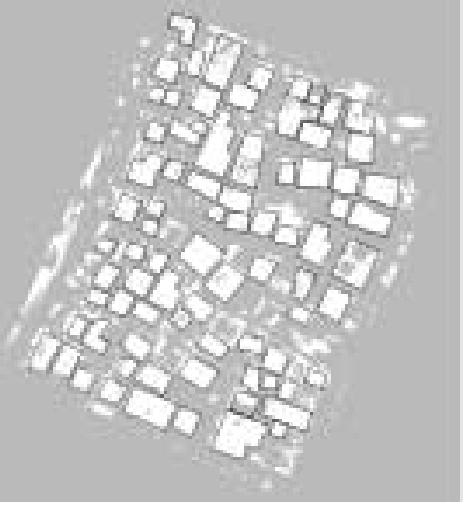

(c)

Figure 4: Results of DSM analysis: (a) DSM (light = high); (b) differences (DSM - DTM) larger than $1.5 \mathrm{~m}$; (c) like (b) after elimination of shadow regions.

\subsection{Attributed Contours}

Edge contours are required in shack reconstruction (cf. Figure 1) for precisely delineating shack boundaries. Related work has shown that in formal urban environments edge contour attributes such as photometry and chromaticity can be successfully employed to connect image contours (edges) for generating object surface hypotheses (Henricsson et al., 1996). Figure 5 shows the (partial) result of contour extraction in the pair of enhanced orthoimages used in the test data set. Very few shacks are completely delineated by contours. Roof-ground interfaces are often weakly extracted. Moreover, many roofs exhibit strong internal edge contours at boundaries between different materials. These pose difficulties for reliable automatic extraction of shack boundaries in the absence of other cues. Edge contours at roof-shadow interfaces are, however, almost without exception reliably extracted in both orthoimages (cf. Figure 5).

\subsection{Shack Delineation}

We now consider the fusion of shack detection results with the extracted attributed contours for shack delineation. While the results of fusing shadow data with DSM blobs are promising for shack detection, we cannot assume that all individual shacks will be accu- 
rately separated, e.g. where shack density is very high blobs may contain multiple shacks, nor that the blob boundaries will accurately delineate shack boundaries at their non-shadow borders. The fusion of shadows with edge contour data presents possibilities for automated and semi-automatic shack extraction. The high contrast between most shack roofs and shadows means that contours defining these interfaces are generally strong and well-defined. In general, these roof-shadow contours can conceivably be used to: (1) verify 3D blobs; (2) refine blob boundaries; and (3) in some cases where blobs are merged to delineate separate adjacent buildings. They can also be employed to hypothesize missing contours, e.g. to guide contour extraction procedures in revisiting local image regions. Most importantly, however, shadow edges can provide the crucial starting point for edge grouping and automated shack extraction.

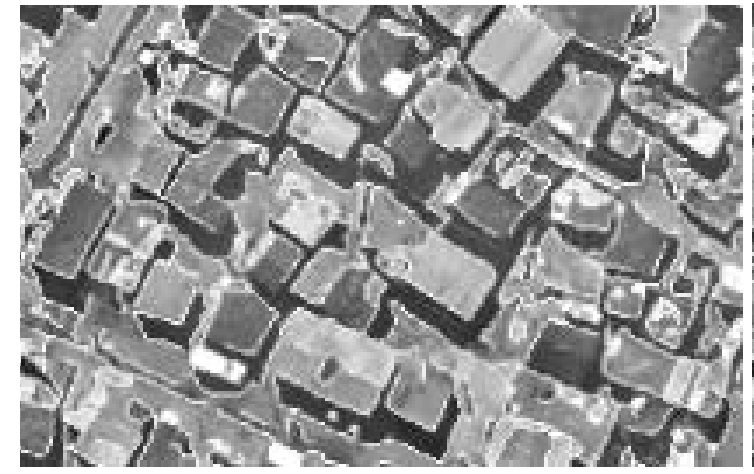

(a)

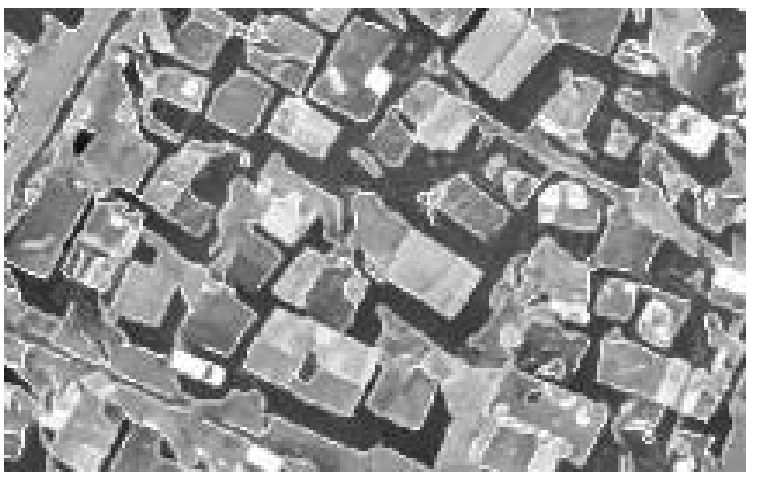

(b)

Figure 5: Example of extracted contours: (a) "left" orthoimage; (b) "right" orthoimage. Shadows show strong delineation.

Two cases for automated shadow contour-based shack extraction are proposed in Figure 6. In Figure 6a two sides of a shack are delineated by shadow contours. These two contours can be used to generate a bounding parallelogram for the 4-sided shack. Note that the shadow direction can either be given manually for a scene or computed automatically from the time and date of image acquisition. Additional contour information can then be used to refine this approximation (Figure 6b). Weak constraints on shack dimensions and contour parallelity and rectangularity can be used to limit the search space. The second case occurs when only one shack side is delineated by a shadow contour (Figure 6c). The DSM blob, the contours and knowledge about min/max shack dimensions are still used to hypothesize one or more bounding parallelograms. In this case, however, there is greater uncertainty in using the extracted contours to refine the delineation. Additional information such as the classification result and edge groupings based on attributes (primarily length, straightness, orientation and to a certain extent colour) may be used to generate hypotheses (Figure 6d). Where automatic extraction fails in the above-mentioned cases, we propose user interaction in the form of pointing to one of the ill-defined corners in the parallelogram as a simple and rapid method to narrow the range of hypotheses to a single satisfactory delineation. 


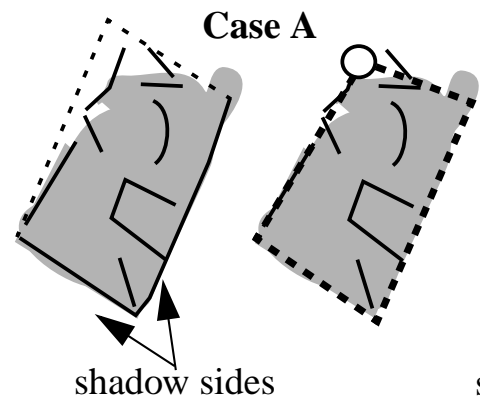

(a) (b)

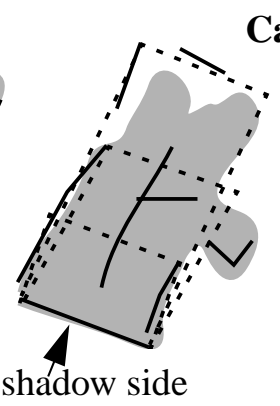

(c)

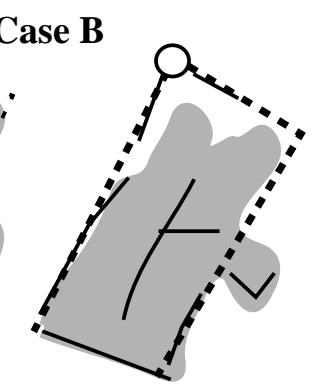

(d)

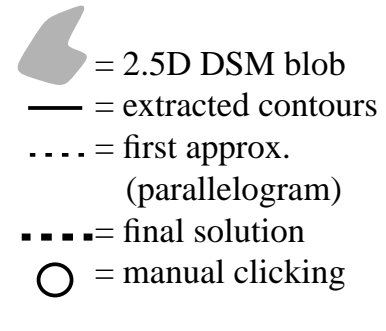

Figure 6: Shack delineation (description in text).

\section{Discussion and Outlook}

A strategy for shack detection and delineation from high resolution still video imagery has been presented. Central to this strategy is the integration of cues in object space. Different cues and their importance for shack reconstruction have been discussed. Preliminary investigations suggest that shack detection can be automatically performed by fusing shadow data with the $2.5 \mathrm{D}$ blobs derived from segmentation of a normalized DSM. These "refined" blobs suffice for a coarse delineation of the individual shacks (if they are not attached), a result which is sufficient for many IS applications (e.g. shack counting). For applications requiring more precise shack delineation we propose using the well-defined edges associated with shadows and other cues to hypothesize shack parallelograms and finally refine them. In difficult cases, user interaction in the form of a single pointing to define a shack corner is foreseen. Emphasis is initially placed on the extraction of geometrically simple 4-sided shacks either automatically or with minimal interaction as these constitute the majority of structures in IS.

In both shack detection and extraction this strategy relies on the presence of shadows in the imagery. To this end, the imagery is best acquired under bright conditions with strong sun inclination angles. We assume that, due to the uniformity of shack heights and general absence of vegetation in IS, shadows on roofs are rare (this does not always hold for IS in tropical countries). A number of cases exist where difficulties in using shadows will occur. Dark roofs may not produce strong shadow contours. Dark materials at roof edges may lead to incorrect parallelogram hypotheses and shape irregularity.

Further investigations are required to verify the use of DSM blobs for shack detection, including:

- Reliability of DSM generation in dense settlements.

- Appropriate methods for cleaning the shadow-refined DSM blobs to remove extraneous blobs.

- The sensitivity of the strategy to the (DSM-DTM) threshold. 
- Use of multispectral classification results in supporting blob definition.

- Use of blobs to predict the number of sides and approximate shape of shacks.

A $2.5 \mathrm{D}$ modeling of shacks is supported by the proposed shack reconstruction strategy. Currently, shack ground plans are inferred from their roof boundaries in a single orthoimage. In future, the fusion of edge contour results from orthoimages of stereopair (cf. Figure 5) will be investigated as a means of resolving (some) ambiguous cases, such as those occurring when only one shack side is defined by shadows. Future work will also include handling of shacks with more than 4 sides, as well as updating of existing shack models.

\section{Acknowledgements}

We acknowledge the contributions of the UrbanModeler team on the Marconi Beam project. This research is supported by the University of Cape Town (URC Entity 453308) and the Foundation for Research Development (Project 2034569). Part of this work was within the project AMOBE at ETH Zurich. We also acknowledge the support and software contribution by Olof Henricsson, ETH Zurich.

\section{References}

Baltsavias E., D. Stallmann, S.O. Mason (1995) Use of DTMs/DSMs and Orthoimages to Support Building Extraction, In: Automatic Extraction of Man-Made Objects from Aerial and Space Images, Gruen A., O. Kuebler, P. Agouris (Eds.), Birkhaeuser Verlag, Basel, pp. 199-210.

Henricsson O., F. Bignone, W. Willuhn, F. Ade, O. Kuebler, E. Baltsavias, S.O. Mason, A. Gruen (1996) Project AMOBE: Strategies, Current Status, and Future Work, Int'1 Archives of Photogrammetry and Remote Sensing, Vol. 31, Part B3, pp. 321 - 330.

Hindson D., J. McCarthy (1994) Defining and Gauging the Problem, In: Here to Stay: Informal Settlements in KwaZulu Natal, Hindson D., J. McCarthy (Eds.), Indicator Press, Durban, pp. 1 - 28.

Li J., S.O. Mason, H. Rüther (1997) Experiences in Automated Shack Extraction Using Multispectral Image Classification, Proc. CONSAS '97, Durban, S. Africa.

Mason S.O., A. Streilein (1996) Photogrammetric Reconstruction of Buildings for 3D City Models, SA J. Surveying \& Mapping, Vol. 23, No. 5, pp. 244 - 262.

Mason S.O., H. Rüther (1997) Managing Informal Settlements Spatially, Proc. ASPRS Annual Meeting, Seattle, USA.

Mason S.O., H. Rüther, J. Smit (1997) Investigation of the Kodak DCS460 for Low-Cost Local Area Mapping, ISPRS J. Photogrammetry \& Remote Sensing (to be published).

UNCHS (1996) Recife International Meeting on Urban Poverty, UNCHS (Habitat). 